\title{
ON A BOOLEAN ALGEBRA OF PROJECTIONS CONSTRUCTED BY DIEUDONNÉ
}

\author{
by H. R. DOWSON \\ (Received 4th July 1968)
}

1. Dieudonné (4) has constructed an example of a Banach space $X$ and a complete Boolean algebra $\widetilde{B}$ of projections on $X$ such that $\widetilde{B}$ has uniform multiplicity two, but for no choice of $x_{1}, x_{2}$ in $X$ and non-zero $E$ in $\widetilde{B}$ is $E X$ the direct sum of the cyclic subspaces $\operatorname{clm}\left\{E x_{1}: E \in \widetilde{B}\right\}$ and $\operatorname{clm}\left\{E x_{2}: E \in \widetilde{B}\right\}$. Tzafriri observed that it could be deduced from Corollary $4(9$, p. 221) that the commutant $\widetilde{B}^{\prime}$ of $\widetilde{B}$ is equal to $A(\widetilde{B})$, the algebra of operators generated by $\widetilde{B}$ in the uniform operator topology. A study of (3) suggested the direct proof of the second property given in this note. From this there follows a simple proof that $\widetilde{B}$ has the first property.

In this connection, the author has shown in (5) that if $\widetilde{B}$ is a complete Boolean algebra of projections on $X$, then when $X$ is a Hilbert space $\widetilde{B}^{\prime}=A(\widetilde{B})$ if and only if $\widetilde{B}$ has uniform multiplicity one, while if $X$ is a Banach space uniform multiplicity one implies that $\widetilde{B}^{\prime}=A(\widetilde{B})$. Therefore in general the reverse implication fails in a Banach space.

2. The reader is referred to (1) and (2) for terminology used in this paper. Next we recall the definition and properties of a class of Banach spaces studied by Halperin (6) and Lorentz (7), (8).

Let $K$ be a compact interval of the form $[0, y]$ where $y>0$. Two nonnegative measurable functions $f_{1}, f_{2}$ on $K$ are said to be equimeasurable if and only if for every $k \geqq 0$

$$
m\left\{x \in K: f_{1}(x) \geqq k\right\}=m\left\{x \in K: f_{2}(x) \geqq k\right\},
$$

where $m(\cdot)$ denotes Lebesgue measure on $R$. For each non-negative measurable function $f$ on $K$, which is finite a.e., the decreasing rearrangement of $f$ is the function $f^{*}$ defined by

$$
\begin{gathered}
f^{*}(0)=\underset{x \in K}{\text { ess. sup }}|f(x)| \\
f^{*}(x)=\sup \{k \geqq 0: m\{y: f(y) \geqq k\} \geqq x\} \quad x \in K, x \neq 0 .
\end{gathered}
$$

$f^{*}$ is continuous on the left. Also $f$ and $f^{*}$ are equimeasurable by construction. Now let $w$ be a positive function which is decreasing and integrable over $K$. The set of equivalence classes of complex measurable functions on $K$ such that $w|f|^{*}$ is integrable forms a Banach space $L_{w}^{1}$ under the norm

$$
\|f\|_{w}=\int_{0}^{y} w|f|^{*} d x
$$


If $m>0$, denote by $f_{m}$ the function equal to $f$ if $|f(x)| \leqq m$ and defined by

$$
f_{m}(x)=\frac{m f(x)}{|f(x)|}, \quad|f(x)|>m .
$$

If $f \in L_{w}^{1},\left\|f-f_{m}\right\|_{w} \rightarrow 0$ as $m \rightarrow \infty$. It follows that the set $B(K)$ of bounded Borel measurable functions on $K$ is norm dense in $L_{w}^{1}$. Now $B(K)$ is a Banach algebra under the supremum norm \|\| . For $f$ in $B(K)$ and $g$ in $L_{w}^{1}$, we have $f g \in L_{w}^{1}$ and $\|f g\|_{w} \leqq\|f\|\|g\|_{w}$. It follows that the map $T_{f}: g \rightarrow f g$ is a bounded linear operator on $L_{w}^{1}$ such that $\left\|T_{f}\right\| \leqq\|f\|$. Moreover the map $f \rightarrow T_{f}$ is a continuous algebra homomorphism of $B(K)$ into an algebra of bounded linear operators on $L_{w}^{1}$.

3. Dieudonné $(4$, p. 10) constructed a compact interval $K=[0, y]$ and functions $w_{1}, w_{2}, w_{3}$ decreasing and integrable over $K$ such that

$$
\begin{gathered}
w_{1}(y)=w_{2}(y)=w_{3}(y)=1 ; \\
\int_{0}^{y} w_{1}^{2} d x=\int_{0}^{y} w_{2}^{2} d x=\int_{0}^{y} w_{3}^{2} d x=+\infty ; \\
\int_{0}^{y} w_{1} w_{2} d x<\infty ; \int_{0}^{y} w_{2} w_{3} d x<\infty ; \int_{0}^{y} w_{3} w_{1} d x<\infty .
\end{gathered}
$$

Let $\tilde{X}$ be the complex Banach space $L_{w_{1}}^{1} \oplus L_{w_{2}}^{1} \oplus L_{w_{3}}^{1}$ under the norm

$$
\left\|\left(f_{1}, f_{2}, f_{3}\right)\right\|=\left\|f_{1}\right\|_{w_{1}}+\left\|f_{2}\right\|_{w_{2}}+\left\|f_{3}\right\|_{w_{3}}
$$

where $f_{i} \in L_{w_{i}}^{1}, i=1,2,3$. Let $X$ be the closed subspace of $\tilde{X}$ consisting of elements $\left(f_{1}, f_{2}, f_{3}\right)$ for which

$$
f_{1}(x)+f_{2}(x)+f_{3}(x)=0 \text { a.e. }
$$

If $\tau \in \Sigma_{K}$, the Borel subsets of $K$, then the map

$$
E(\tau):\left(f_{1}, f_{2}, f_{3}\right) \rightarrow\left(\chi_{\tau} f_{1}, \chi_{\tau} f_{2}, \chi_{\tau} f_{3}\right)
$$

defines a projection on $X$ and the family $\left\{E(\tau): \tau \in \Sigma_{K}\right\}$ forms a $\sigma$-complete Boolean algebra $\widetilde{B}$ of projections on the separable Banach space $X$. Hence $\widetilde{B}$ is a complete countably decomposable Boolean algebra of projections on $X$. $\widetilde{B}$ has uniform multiplicity two $(4$, pp. $7-8)$.

4. Now let $T \in \widetilde{B}^{\prime}$ and let $T(1,0,-1)=(u, v,-u-v)$. We shall show that $v$ is 0 a.e. If not, then since $v$ is integrable over $K$, there would exist a compact subset $\delta$ of $K$ with $m(\delta)>0$ in which $v$ is continuous and non-zero. The hypothesis $T \in \widetilde{B}^{\prime}$ implies that for each function $f$ in $B(K)$

$$
T(f, 0,-f)=(f u, f v,-f u-f v) .
$$

Since $T$ is continuous, the map $A$ of the subspace $\{(f, 0,-f): f \in B(K)\}$ under the $\tilde{X}$-norm into $L_{w_{2}}^{1}$ defined by

$$
A:(f, \dot{0},-f) \rightarrow f v
$$


is continuous. We shall show that this gives a contradiction.

For every $x$ in $\delta$, define

$$
\begin{aligned}
& h(x)=\int_{0}^{x} \chi_{\delta}(t) d t \\
& g(x)=w_{2}(h(x)) .
\end{aligned}
$$

Put $g(x)=0$ for $x$ in $K \backslash \delta$. It follows that $g$ is equimeasurable to the restriction of $w_{2}$ to an interval $\left[0, y^{\prime}\right]$ where $y^{\prime}=m(\delta)$. Hence $g \in L_{w_{1}}^{1}$ and $g \in L_{w_{3}}^{1}$ but $g \notin L_{w_{2}}^{1}$. Since $|v|$ is bounded below in $\delta$ by a positive number $c$, we have $|g v|^{*} \geqq c g^{*}$ and so $g v \notin L_{w_{2}}^{1}$. Now the sequence $\left\{\left(g_{m}, 0,-g_{m}\right)\right\}, m=1,2,3, \ldots$ converges to $(g, 0,-g)$ in $X$ and so by the continuity of the map $A$ the sequence $\left\{g_{m} v: m=1,2,3, \ldots\right\}$ ought to converge to a limit in $L_{w_{2}}^{1}$. However

$$
\left|g_{m} v\right|^{*} \geqq c g_{m}^{*}
$$

This gives a contradiction since the norm of $g_{m}^{*}$ in $L_{w_{2}}^{1}$ becomes arbitrarily large with $m$.

Hence $v=0$. It is now easy to see that $u$ is essentially bounded. If not then for every $m>0$ there would be a measurable set $\tau \subseteq K$ with $m(\tau)>0$ where $|u(x)| \geqq m$. Now

$$
\left\|T\left(\chi_{\tau}, 0,-\chi_{\tau}\right)\right\| \geqq m\left\|\left(\chi_{\tau}, 0,-\chi_{\tau}\right)\right\|
$$

and this contradicts the continuity of $T$. It follows that there is a bounded Borel measurable function $u_{1}$ such that

$$
T(1,0,-1)=\left(u_{1}, 0,-u_{1}\right) .
$$

Similarly there are bounded Borel measurable functions $u_{2}$ and $u_{3}$ such that

$$
\begin{aligned}
& T(0,1,-1)=\left(0, u_{2},-u_{2}\right), \\
& T(1,-1,0)=\left(u_{3},-u_{3}, 0\right) .
\end{aligned}
$$

By the linearity of $T, u_{1}=u_{2}=u_{2}$ a.e. It follows that $\widetilde{B}^{\prime}=A(\widetilde{B})$.

Finally, suppose there is $F \neq 0$ in $\widetilde{B}$ and $x_{1}, x_{2}$ in $X$ such that $F X$ is the direct sum of the cyclic subspaces

$$
M\left(x_{1}\right)=\operatorname{clm}\left\{E x_{1}: E \in \widetilde{B}\right\} \text { and } M\left(x_{2}\right)=\operatorname{clm}\left\{E x_{2}: E \in \widetilde{B}\right\} .
$$

Then $x_{1}, x_{2} \in F X, x_{1} \neq 0$, and $x_{2} \neq 0$, since $\widetilde{B}$ has uniform multiplicity two. It follows readily that there is a projection $P$ in $\widetilde{B}^{\prime}$ with range $M\left(x_{1}\right)$. However, from above every projection in $\widetilde{B}^{\prime}$ lies in $\widetilde{B}$. This contradicts the fact that $\widetilde{B}$ has uniform multiplicity two.

5. It is possible to construct a similar counterexample in which $X$ is uniformly convex. It suffices to take in Section 3

$$
\tilde{X}=L_{w_{1}}^{2} \oplus L_{w_{2}}^{2} \oplus L_{w_{3}}^{2}
$$


under the norm defined by

$$
\left\|\left(f_{1}, f_{2}, f_{3}\right)\right\|=\left[\int_{0}^{y}\left\{w_{1}\left(\left|f_{1}\right|^{*}\right)^{2}+w_{2}\left(\left|f_{2}\right|^{*}\right)^{2}+w_{3}\left(\left|f_{3}\right|^{*}\right)^{2}\right\} d x\right]^{ \pm}
$$

and in Section 4 to define $g(x)=\left(w_{2}(h(x))\right)^{\frac{1}{2}}$ for $x$ in $\delta$.

\section{REFERENCES}

(1) W. G. BADE, On Boolean algebras of projections and algebras of operators, Trans. Amer. Math. Soc. 80 (1955), 345-359.

(2) W. G. BADE, A multiplicity theory for Boolean algebras of projections on Banach spaces, Trans. Amer. Math. Soc. 92 (1959), 508-530.

(3) J. Dieudonne, Sur la bicommutante d'une algèbre d'opérateurs, Portugal. Math. 14 (1955), 35-38.

(4) J. Dieudonnt, Champs de vecteurs non localement triviaux, Arch. Math. 7 (1956), 6-10.

(5) H. R. Dowson, On the commutant of a complete Boolean algebra of projections, Proc. Amer. Math. Soc. 19 (1968), 1448-1452.

(6) I. Halperin, Function spaces, Canad. J. Math. 5 (1953), 273-288.

(7) G. LoRenTZ, Some new functional spaces, Ann. of Math. (2) 51 (1950), 37-55.

(8) G. LoRentz, On the theory of spaces $\Lambda$, Pacific J. Math. 1 (1951), 411-429.

(9) L. TZAFriri, On multiplicity theory for Boolean algebras of projections, Israel J. Math. 4 (1966), 217-224.

UNIVERSITY OF ILLINOIS

URBANA, ILLINOIS

AND

UNIVERSITY OF GLASGOW 